Observer et comprendre les populations d'usagers de l'information scientifique et technique, c'est se préparer à leur offrir des services adaptés. Cette étude proposée par Gérald Kembellec offre des éclairages sur les usages en sciences humaines et sociales, ainsi qu'en sciences dures. L'auteur observe également les personnes qui assistent les enseignants chercheurs dans leur production scientifique : documentalistes et bibliothécaires. Il y dégage ensuite des réflexions et préconisations pour orienter les fonctionnalités des services numériques pour la recherche de littérature scientifique.

\title{
La médiation technologique autour des pratiques rédactionnelles et bibliographiquesen milieu universitaire français
}

\author{
Gérald KEMBELLEC \\ gerald.kembellec@cnam.fr
}

\section{Laboratoire Paragraphe \\ Université Paris 8 \\ 2 rue de la Liberté \\ 93526 Saint-Denis Cedex}

\author{
INTD \\ Conservatoire National des Arts et Métiers \\ case courrier 1D6P40 \\ 77003 Paris
}

Cette étude exploratoire réunit des données autour des pratiques rédactionnelles et documentaires dans les milieux scientifiques universitaires français. Nous faisons émerger un cahier des charges fonctionnel pour les systèmes de recherche d'informations (SRI) scientifiques et techniques. Placer les pratiques des usagers comme fondement de la conceptualisation d'un système d'information (SI)est un prérequis pour envisager une "valeur ajoutée globalisée »[16]. Selon de Kaenel et Iriarte, "les dernières évolutions du web, avec l'entrée en jeu de XML, des nouveaux usages et nouveaux outils, ainsi que le déplacement du centre de gravité qui s'est fortement rapproché des utilisateurs, ouvrent de nouvelles voies et de nouveaux champs d'application pour les catalogues en ligne»[8].

Nous organisons notre étude autour de plusieurs axes techniques et sociologiques dans le contexte pluridisciplinaire des sciences de l'information et de la communication (SIC) et proposons de faire émerger les habitudes technologiques bibliographiques associées aux pratiques rédactionnelles des universitaires. Notre attention se focalise particulièrement sur les doctorants, documentalistes et enseignants chercheurs. Ces observations sont établies sociologiquement pour les usagers en fonction de leur niveau d'expertise et de leurs centres d'intérêt scientifiques.

\section{PROBLÉMATIQUE}

Bermèset Martin déclarent qu'une «collection numérique ne peut pas être appréhendée directement. Elle requiert une médiation technique [...] entre l'usager et la collection "[3].L'objet de la présente étude est de déterminer des pratiques d'usagersde l'information scientifique et technique (IST) au travers de l'observation de leurs choix techniques. Ainsi, munis d'informations relatives aux usages, nous pouvons synthétiser les besoins en terme de médiation technique. Ces données permettront ultérieurement de créer un outil répondant aux besoins techniquespar une "urbanisation » du SIad hoc[7].Nous entendons, par le terme d'urbanisation, les fonctionnalités 
communicationnelles entre les logiciels de l'utilisateur et le SRI.Cela se concrétise par des fonctions «ExtractTransformLoad »capables de fournir le flux informationnel dans le formatattendu par un processus automatisé[17], en plus de celui compréhensible par l'humain. Il n'est, en effet, pas possible de modéliser un SRI et, plus généralement, un SI ${ }^{1}$, sans se référer directement aux technologies de l'information[9]. Cette étude permet également de spécifier des préconisations d'usage, profitablesprincipalement aux universitaires novices, pour les guider dans leurs pratiques bibliographiques. De plus, elle peut être exploitéepour mesurer l'adéquation technique des SRI en IST avec les outils communément usités par les communautés de chercheurs.

Pour mieux couvrir les besoins technologiques en matière informationnelle, il est possible d'envisager des points d'accès multiples à la même information scientifique et technique, ce qu'illustrent la base bibliographiqueDBLP2 ou la plate-forme de rechercheIsidore $^{3}$ [12]. De plus, les autres facteurs inhérents au web de données,comme l'interopérabilité,doivent être respectés. Il s'agit de l'exposition des métadonnées grâce aux formats de fichiers bibliographiques, aux technologies RDFa et microformats[11], ou encore à celle du Context Object in Span(COinS). Ces formats sont déjà exploités par nombres d'éditeurs scientifiques, d'archives ouvertes ou de moteurs scientifiques. Selon l'axe de lecture scientifique et les choix techniques en matière de pratique bibliographique, les utilisateurs pourraient alors avoir un accès adapté à leurs attentes.

Pour les doctorants et les enseignants chercheurs principalement, nous avons tenu à distinguer les sciences, techniques et médecine (STM), dites "sciences dures", des sciences humaines et sociales (SHS). Cette distinction est importante en terme d'usages, car les outils et protocoles de production scientifiquequi en découlent diffèrent. En sciences dures, il est courant que la demande des revues et conférencesimpose un protocole d'écriture précis. Les documents doivent souvent être rédigés en employant le format LaTeX, ce qui induit l'usage du format bibliographique BibTeX. En sciences humaines et sociales, les usages sont différents. Nous allons définir dans quelle mesure ces usages se singularisent selon les profils des universitaires.

Les divergences entre les deux groupesne s'arrêtent pas à la production normée de documents. Les usages en terme d'outils varient également, notamment en matière de stockage des notices bibliographiques.

D'autres études précédentes donnent une idée des logiciels et formats les plus usités pour la gestion bibliographique.En 2009, Masur présentait les logiciels proposés par les SCDdes universités suisses[10] et concluait que le public universitaire connaissait encore assez mal les LGRB4. Pour formuler nos questions en ce domaine, nous nous sommes basés sur l'étude de Carole Zweifelqui présentaitseptdes principaux logiciels de gestion bibliographique[19]. Nous avons sélectionné parmi ces logiciels ceux dits "autonomes $»^{5}$, auxquels nous avons ajouté RefBase qui est parfois installé par les services informatiques universitaires comme outil de partage bibliographique pour une équipe ou un laboratoire. Nous avons complétéce panel avec les deux outils propriétaires parmi les plus connus :EndNote et RefWorks. Le questionnaire permetau répondant de cocher le (ou les) outil(s) de gestion bibliographique dont il se sert le plus parmi six des plus connus. Il a également la possibilité de proposer un outil alternatif s'il le désire.

1 Définition de système d'information : «Processus qui collectent des données structurées conformément aux besoins [...], qui stockent, traitent et distribuent l'information »[2].

2 DBLP offre 2 interfaces : une « humanreadable » et une d'interrogation SparQL [5]

3 Isidore propose 3 interfaces : http://rechercheisidore.fr/ pour l'interface de recherche intuitive, http://rechercheisidore.fr/api pour l'interconnexion avec les outils logiciels et http://rechercheisidore.fr/sparql pour les adeptes du langage de requête SparQL[5].

4Acronyme de logiciel de gestion de références bibliographiques, imputé à Zweifel

5 Qui ne nécessitent pas l'installation d'un serveur hypertexte et d'une base de données. 


\section{MÉTHODOLOGIE D’ENQUÊTE}

Typologie et méthode de diffusion du questionnaire

Nous avons établi un plan de sondage en 6 sections, de la manière suivante :

1. Section de filtrage pour établir des profils scientifiques avec des questions fermées (statut, expérience, science étudiée).

2. Partie sur le contexte technique d'écriture scientifique; section composée de questions fermées (système d'exploitation et logiciels connus et employés).

3. Section plus spécifique sur les pratiques bibliographiques avec des questions fermées et semi-ouvertes.

4. Section sur l'impact du « libre » et du« gratuit »sur le choix de l'utilisateuravec des questions fermées et semi-ouvertes.

5. Partie sur les priorités dans le choix d'un logiciel bibliographique, composée de questions semi-ouvertes.

6. Question semi-fermée sur le choix des sources d'informations scientifiques.

Typologie des questions ${ }^{6}$ :

Questions fermées : 1 choix parmi 3 ou 4

Questions semi-fermées : 1 ou plusieurs choix parmi plusieurs

Questions semi-ouvertes : 1 ou plusieurs choix parmi plusieurs ou la possibilité de

fournir sa propre réponse

Population cible et panel

La question de la représentativité d'un panel de sondés est cruciale pour la crédibilité d'une enquête. Nous ne faisons pas intervenir ici de questions sur l'âge ou le sexe, ce qui nous semble sans objet.

À partir d'une vingtaine de personnes par échantillon de la population observée, des tendances émergent. Cependant, plus le public est hétérogène, plus il faut élargir le panel[13]. Notre étude, extrêmement ciblée, est principalementquantitative. Dans notre cadre, contrairement aux sondages d'opinion, il n'est pas nécessaire d'interroger beaucoup d'individus de chaque catégorie. Pour chaque échantillon étudié, 20 à 25 personnes suffisent pour obtenir de bons résultats[18].Quand à la marge d'erreur, elle se situe, pour une population entre 100 et 200 personnes et dans le cadre de ce type de sondage, entre 3.1 et 4.4 points[14].

Cette enquête a été menée sur une période de 15 jours en décembre 2011. Le formulaire, mis en ligne sur Google Formulaires,a été soumis sur la base du bénévolat et de l'anonymat. Nous évaluons le nombre de personnes contactées par courriel à 9000 personnes entre les grosses listes de diffusion (ADBS : 7400 et $\mathrm{EGC}^{7}: 1000$ ) et les petites listes d'écoles doctorales et de laboratoires d'universités ou de grands établissements (600).

\section{LES RÉSULTATS}

Taux de retours et répondants

Le panel de personnes ayant répondu est constitué de 195 participants soit 2,17 \% des contactés.La distribution est la suivante :

- 54 enseignants chercheurs dont 16 en STM, 36 en SHS et 2 dans d'autres domaines.

- 98 doctorants dont 11 en STM et 79 en SHS, 8 dans d'autres domaines.

6 Pour encourager les destinataires de l'étude à répondre au questionnaire, nous avons volontairement simplifié le questionnaire en réduisant la typologie des questions. Ainsi, le temps des sondés est épargné au maximum.

7 EGC : Association Extraction et gestion des connaissances, http://www.egc.asso.fr 
- 28 personnels de SCD

- 4 postdoctorants

- 11 personnes hors de ce classement (par exemple des ingénieurs de recherche).

\begin{tabular}{|l|r|r|}
\hline & Doctorant début de thèse & Doctorant fin de thèse \\
\hline Sciences humaines et sociales & 34 & 45 \\
\hline Sciences dures & 5 & 6 \\
\hline Autres & 5 & 3 \\
\hline
\end{tabular}

Tableau 1. Répartition des doctorants par expérience et domaine de recherche

Nous n'étudions pas en détail le statut de postdoctorant, car le segment interrogé ne présente pas un corps suffisamment élevé pour être représentatif. Nous avons segmenté le statut de doctorant (Tableau 1) pour spécifier l'expérience en début (44 personnes) et fin de thèse ( 54 personnes). Pour caractériser les profils utilisateurs, nous avons encore dégagé 4 sous-ensembles principaux en fonction de l'expérience et du domaine de recherche. Il sera pertinent de faire une distinction sur l'évolution des usages technologiques liés aux pratiques bibliographiques pour les doctorants en SHS du fait d'un grand nombre de participants.

Pour chaque question, nous présenterons les résultats de manière générale puis détaillée pour les SHS et STM. Enfin, nous présentons les résultats pour les documentalistes.

\section{Usages d'outils de productions écrites en sciences}

Dans cette partie, nous établissons le profil technique d'un usagerrelativement à l'écriture scientifique. Ce profil est composé de l'ensemble des choix qu'un usager effectue en terme d'outils informatiques. Il peut s'agir de système d'exploitation ou de logiciel, ou encore de normalisation de fichiers.

Pour écrire un document scientifique, il existe deux options : le traitement de texte ou le compilateur de texte.Les traitements de texte sont des éditeurs graphiques comme Word ou OpenOffice. Un compilateur de texte, comme LaTeX, est un outil, généralement non graphique, qui permet de faire une mise en page professionnelle de documents complexes.

Dans le cadre de l'écriture scientifique, l'usage d'éditeurs graphiques comme Word et OpenOffice est largement privilégié. Cependant, pour le segment des enseignants chercheurs, l'écart entre le pourcentage d'usagers de compilateurs et de traitement de textes est très réduit. Dans les autres groupes, l'usage des compilateurs de texte est quasi nulsauf pour les doctorants en fin de thèse et les ingénieurs (un peu plus de $18 \%$ dans les deux cas).

\begin{tabular}{|l|r|r|r|r|r|r|}
\hline $\begin{array}{c}\text { Outil de } \\
\text { production de } \\
\text { documents }\end{array}$ & $\begin{array}{c}\text { Doctorants } \\
\text { en début de } \\
\text { thèse }\end{array}$ & $\begin{array}{c}\text { Doctorants } \\
\text { en fin de } \\
\text { thèse }\end{array}$ & $\begin{array}{r}\text { Post- } \\
\text { doctorants }\end{array}$ & $\begin{array}{c}\text { Enseignants } \\
\text { chercheurs }\end{array}$ & $\begin{array}{c}\text { Personnels } \\
\text { de SCD }\end{array}$ & $\begin{array}{c}\text { Autres } \\
\text { Ingénieurs }\end{array}$ \\
\hline TeX ou LaTeX & $10,0 \%$ & $18,5 \%$ & $50,0 \%$ & $46,3 \%$ & $3,6 \%$ & $18,2 \%$ \\
\hline $\begin{array}{l}\text { Éditeur } \\
\text { graphique }\end{array}$ & $90,0 \%$ & $81,5 \%$ & $50,0 \%$ & $53,7 \%$ & $92,8 \%$ & $71,7 \%$ \\
\hline Autre & $0,0 \%$ & $0,0 \%$ & $0,0 \%$ & $0,0 \%$ & $3,6 \%$ & $9,1 \%$ \\
\hline
\end{tabular}

Tableau 2. Outil de production de documents par profil d'utilisateurs.

En sciences humaines, l'usage du compilateur évolue, avec l'expérience, de presque nulen début de thèseàfaible pour les enseignants chercheurs.

\footnotetext{
${ }^{8}$ Lapopulation de post-doctorants est trop faible pour être considérée comme représentative.
} 


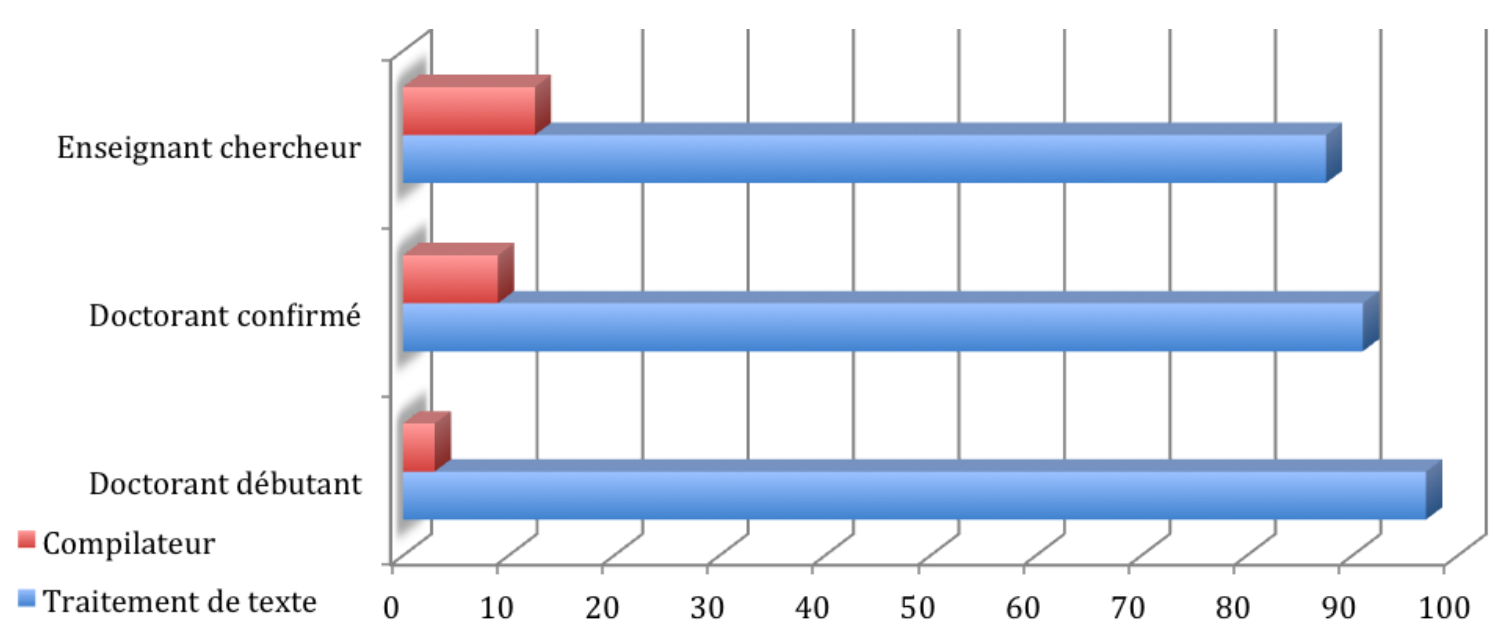

Figure 1. Méthode d'écriture en SHS selon l'expérience

L'emploi d'un compilateur est plus développé dans lessciences dures, notamment pour les doctorants en fin de thèse (voir Figure 2). Cette partie de la population rédige son mémoire de thèse avec des équations et une bibliographie complexes. Entre ces considérations techniques et des pratiques culturelles universitaires très orientées vers LaTeX, l'usage d'un compilateur de texte est presque obligatoire pour une thèse de doctorat en sciences dures. Cette population édite les fichiers LaTeX de la manière suivante : éditeur à compilation intégrée (TexWorks, Emacs, etc.) : $72 \%$; éditeur basique (Vi, Ed, Notepad, etc.) : $15 \%$; autre : $22 \%$.

Les propositions, pour les habitués de TeX/LaTeX ayant coché la valeur «autre », sont les suivantes:Kile ${ }^{9}, \mathrm{LyX}^{10}$ (4 personnes l'ont proposé dont 3 en sciences humaines), TeXnicCenter ${ }^{11}$, usbTex ${ }^{12}$ et Eclipse avec le plug-inTeXlipse.

Chez les documentalistes et bibliothécaires, l'usage du traitement de texte (97\%) est presque unanime.

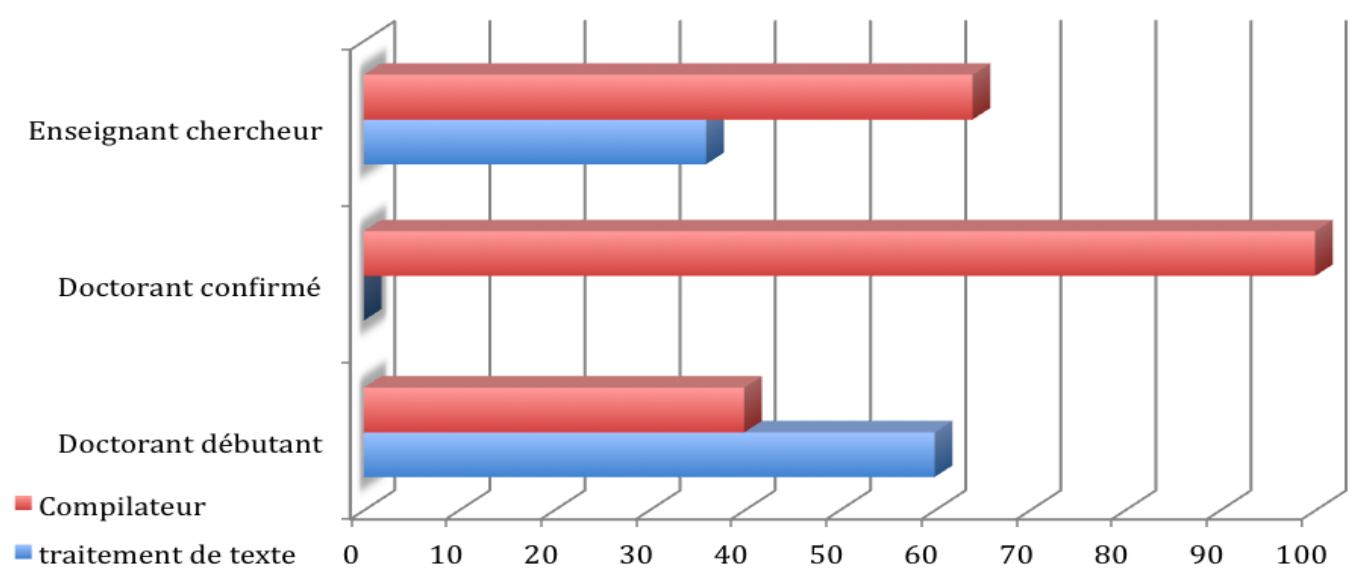

Figure 2. Méthode d'écriture en sciences dures selon l'expérience

Les usages et formats de bibliographie

Pour mieux cerner les usages scientifiques en matière de bibliographie, nous avons demandé à la population de sondés leurs pratiques. Intègrent-ils leurs citations et

\footnotetext{
9Kile est un éditeur graphique de TeX/LaTeX disponible sur Mac OS, Linux et Microsoft Windows : http://kile.sourceforge.net/ et http://www.framasoft.net/article2827.html

10 Environnement TeX avec une interface entièrement graphique utilisable à la souris http://www.framasoft.net/article1001.html

11 Environnement intégré de développement TeX en C++ sous Windows http://www.framasoft.net/article1429.html

12 Environnement TeX complet transportable sur clé USB : http://www.framasoft.net/article4641.html
} 
bibliographies manuellement ou lesgénèrent-ils(que ce soit grâce au compilateur LaTeXouàleur outil de traitement de texte) ?

Il semble que, de manière globale (se reporter à la Figure 3), l'introduction des références et bibliographies se fasse encore principalement d'une manière manuelle dans le texte (37\%). Pour ceux qui utilisent un fichier bibliographique séparé, 19\% l'enrichissent manuellement et $18 \%$ s'appuient sur un logiciel bibliographique. En tenant compte des $25 \%$ de personnes qui exploitentl'outil interne à leur traitement de texte, $63 \%$ des sondés en milieu universitaireprofitentd'une fonction automatisée de gestion de bibliographie. Ce chiffre mérite d'approfondir l'analyse despratiques liées à ces outils pour mieux mettre en adéquation les services des SRI avec les besoins des usagers.

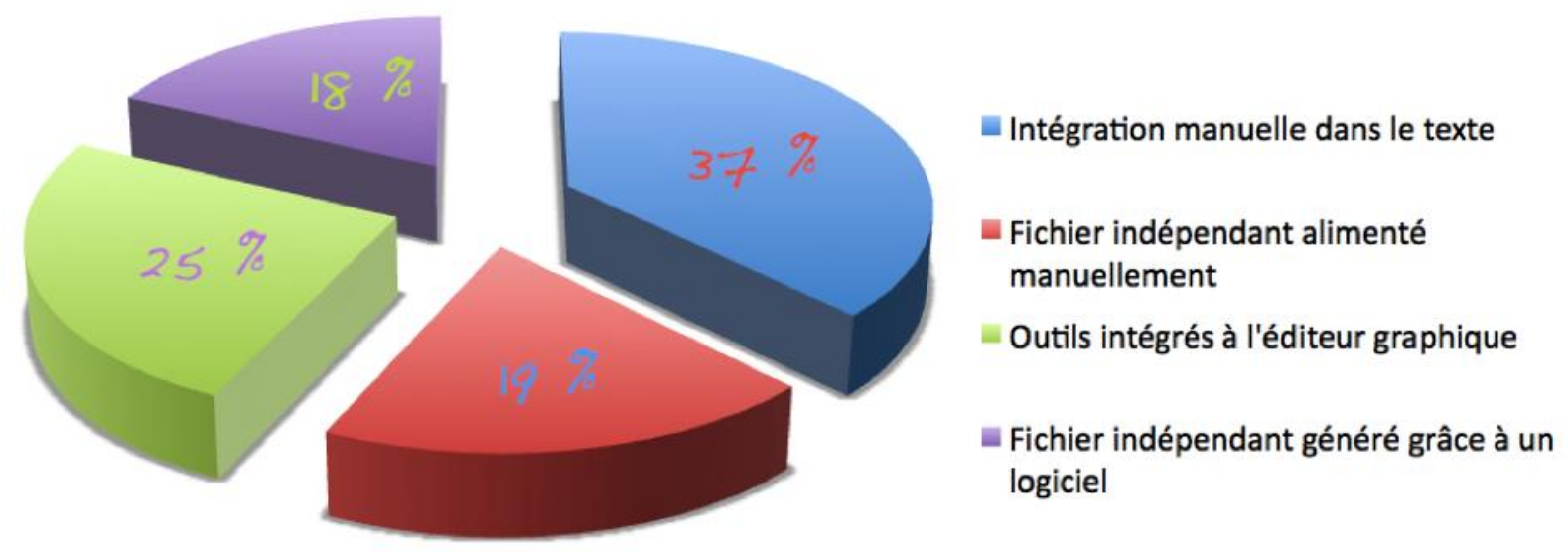

Figure 3. Méthode d'intégration bibliographique dans un document pour l'ensemble de la population (valeurs arrondies à l'unité).

Si l'on répartit ces résultats par sciences, $20 \%$ des sondés en sciences dures intègrent manuellement leurs références dans le texte pour $47 \%$ en sciences humaines. La population la plus éloignée de cet usage est celle des personnels de SCD puisque seuls $17,86 \%$ des sondés intègrent leurs références manuellement dans le texte.

Nous avons ensuite interrogé la population sur les formats de bibliographie utilisés dans le cadre de la rédaction de documents scientifiques, thèse ou rapports techniques. Plusieurs réponses, personnalisées si les répondants le souhaitaient, étaient admises.L'analyse des chiffres avant répartition indique que le format bibliographique le plus usitéest BibTeX. Cependant, $45 \%$ de l'échantillon n'entretiennentpas de fichier séparé pour gérer les bibliographies. Nous avons également eu des réponses alternatives très pointues comme: Zotero RDF13, APA ${ }^{14}$ (2 chercheurs), MARC $^{15}$ (1 documentaliste).

En SHS, quasiment les deux tiers des sondés $(61,74 \%)$ ne maintiennent pas de fichier formaté pour la gestion de leur bibliographie. Parmi ces derniers,29,58\% se servent néanmoins des fonctions bibliographiques intégrées à leur traitement de texte. Pour ceux qui utilisentun fichier, c'est principalement BibTeX qui a leur faveur pour le formatage, à presque $50 \%$. Pour le reste, $23,91 \%$ des utilisateurs de fichiers bibliographiques formatés se servent de XMLOpenDocument et environ $20 \% \mathrm{du}$

13 Format XML de description de notices bibliographiques généré par Zotero, plug-in de Firefox.

14 L'APA est un format de présentation pour les références bibliographiques, notes de bas de page, citations dans les publications. Ce format est défini par l'American Psychological Association [1].

15 Format de données bibliographiques de la Bibliothèque du Congrès, http://www.loc.gov/marc/bibliographic. 
formatRIS. À la marge, $9 \%$ des personnes interrogées, dans ce segment, pratiquent d'autres formats.

Parmi les 34doctorants en début de thèse de SHS, 14,7 \% utilisent le format BibTeX, ce qui est étonnamment élevé, si l'on prend en compte le faitque beaucoup n'ont pas encore commencé la rédaction de leur manuscrit et que ce format est complexe à appréhender. Dans cette même population,3,22 \% adoptent le format RIS et seulement $5,88 \%$ le format XML OpenDocument, plus adapté aux éditeurs graphiques, notamment Word depuis sa version 2007.

\begin{tabular}{|l|r|r|r|r|r|r|r|}
\hline $\begin{array}{l}\text { Formats utilisés par } \\
\text { la population SHS }\end{array}$ & BibTeX & RIS & Ovid & $\begin{array}{l}\text { XML } \\
\text { Open } \\
\text { Document }\end{array}$ & Autre & NSP & Aucun \\
\hline Utilisation unique & $13,00 \%$ & $5,20 \%$ & $0,00 \%$ & $6,90 \%$ & $2,60 \%$ & - & - \\
\hline Utilisation multiple & $5,20 \%$ & $3,50 \%$ & $0,00 \%$ & $2,60 \%$ & $0,90 \%$ & - & - \\
\hline \%de la population SHS & $18,26 \%$ & $8,69 \%$ & $0,00 \%$ & $9,56 \%$ & $3,48 \%$ & $1,50 \%$ & $61,74 \%$ \\
\% de la population & $47,73 \%$ & $18,75 \%$ & $0,00 \%$ & $23,91 \%$ & $9,09 \%$ & - & - \\
$\begin{array}{l}\text { SHS utilisant au } \\
\text { moins un format }\end{array}$ & & & & & & & \\
\hline
\end{tabular}

Tableau 3. Formats bibliographiques utilisés en SHS

Pour les 45doctorants de SHS en fin de thèse, 30 n'adoptent pas de format bibliographique, soit les 2/3. Pourtant,avec Word par exemple, il est possible d'introduire les centaines de notices bibliographiques d'une thèse et d'y faire référence au travers de la base de données intégrée (XML OpenDocument).Comme $80 \%$ des doctorants rédigent leur thèse et leurs articles grâce à un traitement de texte, on pourrait s'attendre à un usage plus important de ce format adapté.

En ce qui concerne le sous-ensemble des enseignants chercheurs et postdoctorants en SHS (18 individus), 55,56 \%ne font usaged'aucun fichier formaté de bibliographie. Pour ceux qui en font usage, 22,22\% choisissent BibTeX $^{16}, 11,11 \%$ RIS et 11,11\% XML OpenDocument.

\begin{tabular}{|l|r|r|r|r|r|}
\hline $\begin{array}{l}\text { Formats utilisés par la } \\
\text { population de sciences dures }\end{array}$ & BibTeX & \multicolumn{1}{l|}{ RIS } & Ovid & $\begin{array}{l}\text { XML } \\
\text { OpenDocument }\end{array}$ & \multicolumn{1}{l|}{ Aucun } \\
\hline Utilisation unique & $72,40 \%$ & $3,40 \%$ & $1,70 \%$ & $0,00 \%$ & - \\
\hline Utilisation multiple & $6,90 \%$ & $5,20 \%$ & $1,70 \%$ & $6,90 \%$ & - \\
\hline $\begin{array}{l}\text { \% de la population sc. dures } \\
\text { \% de la population sc. dures } \\
\text { utilisant au moins un format }\end{array}$ & $79,31 \%$ & $8,62 \%$ & $3,45 \%$ & $6,90 \%$ & $13,79 \%$ \\
\hline
\end{tabular}

Tableau 4. Formats bibliographiques utilisés en Sciences dures

L'usage d'un fichier séparé pour la bibliographie est mieux ancré dans la culture scientifique. Seuls $13,79 \%$ de ce groupe n'utilisent aucun fichier bibliographique.Parmi ceux-ci, unepersonne seulementsur quatre intègre ses citations manuellement. Les autres exploitent les fonctionnalités prévues à cet effet par leur traitement de texte. Cependant, en sciences dures, le format BibTeX fait consensus. 72,40\% de la totalité du groupel'ontadoptéexclusivement et $92 \%$ de ceux qui se servent d'au moins un format de fichierchoisissent BibTeX. Seulle format RIS est également citécomme format unique

\footnotetext{
${ }^{16}$ Notons la publication récente d'un livre[15],qui semble indiquer une possible évolution de l'usage de LaTeXen SHS.
} 
par 3,40 \% de la population. Nous pouvons remarquer(tableau 4) un usage marginal du XML OpenDocument (6,90 \%) comme format secondaire.

\begin{tabular}{|l|r|r|r|r|r|r|}
\hline $\begin{array}{l}\text { Formats utilisés par la population } \\
\text { de personnels de SCD }\end{array}$ & BibTeX & RIS & \multicolumn{1}{l|}{ Ovid } & $\begin{array}{l}\text { XML } \\
\text { Open } \\
\text { Document }\end{array}$ & Autre & Aucun \\
\hline Utilisation unique & $7,10 \%$ & $25,00 \%$ & $0,00 \%$ & $7,10 \%$ & $3,60 \%$ & - \\
\hline Utilisation multiple & $21,40 \%$ & $21,40 \%$ & $7,10 \%$ & $17,90 \%$ & $0,00 \%$ & - \\
\hline \%de la population de SCD & $28,57 \%$ & $46,43 \%$ & $7,14 \%$ & $25,00 \%$ & $3,57 \%$ & $28,57 \%$ \\
$\begin{array}{l}\text { \%de la population de SCD } \\
\text { utilisant au moins un format }\end{array}$ & $40,00 \%$ & $65,00 \%$ & $10,00 \%$ & $35,00 \%$ & $5,00 \%$ & - \\
\hline
\end{tabular}

Tableau 5. Formats bibliographiques utilisés par les documentalistes et bibliothécaires en SCD.

Les documentalistes et bibliothécaires exploitentun large panel de formats de fichiers bibliographiques (tableau 5). Parmi les personnes qui manipulentau moins un format de fichier,65 \% citent leformat RIS et $40 \%$ LaTeX(l'exploitation de plusieurs formats par une même personne expliquedes totaux supérieurs à $100 \%$ ) ; quant au format XML OpenDocument, il remporte $35 \%$ des suffrages. Ceux qui n'utilisent pas de fichier bibliographique formaté se servent des outils intégrés à leur traitement de texte $75 \%$ des cas). Sur l'ensemble de notre panel, seulement $7,14 \%$ des sondés intègrent leurs références bibliographiques manuellement dans les écrits. Cela confirme une culture « métier », bien ancrée dans les pratiques.

Usages et pratiques de logiciels de gestion de références bibliographiques

LGRB ou pas?

S'agissant des logiciels de gestion de références bibliographiques (LGRB), $56 \%$ des répondants enexploitentun, $38 \%$ n'en utilisent paset,pour $6 \%$, cette question est sans objet. Ces résultats se retrouvent dans toutes les sciences.

En SHS, seuls 53,91 \% des répondants emploient au moins un LGRB. Cette question est sans objet pour $7,83 \%$ de l'échantillon.Ces derniersutilisent plus volontiers les fonctionnalités du traitement de texte $(20,45 \%)$, mais, plus souvent, intègrent les éléments bibliographiques sans aucuneassistance logicielle (75\%).Ceux qui ne sont dans aucun des cas cités précédemment entretiennent d'une façon manuelle un fichier bibliographiquedans un format compatible avec leur outil rédactionnel.

En STM,l'exploitation d'au moins un logiciel de gestion bibliographique concerne $53,45 \%$ des répondants et cette question est sans objet pour 5,17\%. De manière générale, ceux qui n'ont pas recours à un LGRB intègrent leurs références manuellement dans un fichier bibliographique formaté (58,46\%)ousaisissent directement les références dans le texte $(29,17 \%)$. Ils ne sont que $8,33 \%$ de ceux qui n'utilisent pas de LGRB à profiter des fonctionnalités dédiées d'un traitement de texte.

Pour la population de répondants documentalistes et bibliothécaires, la réponse a été presque unanimement affirmative $(96,43 \%)$.

\section{Quels LGRB ?}

Au sein des outils de type LGRB, Zotero est largement plébiscité. Parmi les progiciels, EndNote de Thomson Reuters, bien que payant, est également très largement cité $(29,38 \%$ de la population). Il arrive que les deux logiciels soient employés conjointement $(12,84 \%$ de l'ensemble du panel). Tous les pourcentages proposés pour la ventilation des usages de LGRB (voir Tableau 6. Ventilation de l'usage des LGRB) s'entendent par rapport au nombre de personnes utilisatriceset nonpar rapport à la 
totalité de la population de référence. Parmi les logiciels signalés par les sondés sous la catégorie "autres", nous avons eu la surprise de voir des personnes recouriràXMind, logiciel de "concept map », pour noter les références bibliographiques. Cette pratique peut surprendre, mais cela permet de visualiser les rapports de coécritures et de citations entre documents. Les autres logiciels proposés sont ReferenceManager (cité 2 fois par des personnels de SCD) et le logiciel libre Pybliographer ${ }^{17}$ (cité 1 fois). La version Web d'EndNote a été également citée 2 fois. Deux personnes en sciences dures, un enseignant-chercheur et un postdoctorant ont déclaré utiliser le portail ACM comme gestionnaire de bibliographie. Après vérification, le portail ACM propose une option soumise à abonnement «My Binders » pour stocker des hyperliens vers les notices des articles repérés sur le portail. Deux personnes tirent profit d'HAL, portail de dépôt d'articles en ligne du CNRS, pour gérer des notices bibliographiques.

En SHS, Zotero est très largement usité (presque $63 \%$ des utilisateurs de LGRB en SHS). Vient ensuite EndNote (presque $30 \%$ ), parfois coupléavec Zotero. Cela s'explique par le fait que ces populations peuvent tirer parti deZoterode deux manières: comme un logiciel uniquedont l'usage va de la détection, sur les sites compatibles, de notices bibliographiques à l'export dans Word ou OpenOffice en passant par le stockage et la gestion de la bibliographie; ou comme module permettant de détecter les documents scientifiques, dontles notices sont exportées dans EndNote qui se chargera ensuite de la gestion et de l'intégration dans le traitement de texte.

L'écriture de documents en sciences dures nécessite souvent l'usage du format BibTeX. Cela explique que JabRef arrive en tête des logiciels avec presque $30 \%$ des utilisateurs de LGRB en sciences dures.Mendeley $(38,71 \%)$ et Zotero $(32,26 \%)$ sont également largement employés. Pour les mêmes raisons et dans les mêmes conditions qu'en SHS, Zotero est parfois utilisé en collaboration avec Mendeley et JabRef. Le premieroffre la possibilité de communiquer avec Word grâce à un plug-in, alors que le secondse spécialise dans la création et la gestion de fichiersBibTeX.JabRef, par exemple, permet de détecter les erreurs de typage dans un fichier BibTeX. Le chercheur s'épargnera ainsi une fastidieuse étape de débogage à la compilation de la bibliographie du document.

\begin{tabular}{|l|r|r|r|r|}
\hline $\begin{array}{l}\text { LGRB } \\
\text { utilisé }\end{array}$ & \% global de la population & \% en SHS & \% en sc. dures & En SCD \\
\hline Zotero & $54,13 \%$ & $62,90 \%$ & $32,26 \%$ & $88,89 \%$ \\
\hline JabRef & $20,18 \%$ & $9,68 \%$ & $45,16 \%$ & $14,81 \%$ \\
\hline RefWorks & $0,92 \%$ & $1,61 \%$ & $3,23 \%$ & $0,00 \%$ \\
\hline BibDesk & $6,42 \%$ & $3,22 \%$ & $16,13 \%$ & $0,00 \%$ \\
\hline Mendeley & $21,10 \%$ & $11,29 \%$ & $38,71 \%$ & $25,93 \%$ \\
\hline EndNote & $29,36 \%$ & $33,87 \%$ & $16,13 \%$ & $40,74 \%$ \\
\hline Bibus & $0,92 \%$ & $0,00 \%$ & $0,00 \%$ & $3,70 \%$ \\
\hline RefBase & $0,00 \%$ & $0,00 \%$ & $0,00 \%$ & $0,00 \%$ \\
\hline Autres & $11,01 \%$ & $1,61 \%$ & $12,90 \%$ & $3,70 \%$ \\
\hline
\end{tabular}

Tableau 6. Ventilation de l'usage des LGRB

La seule personne en SCD de notre panel qui ne se sert pas de LGRB exploite les notices Marc individuelles générées par le système de gestion de la bibliothèque. Dans l'ensemble, les personnels de SCD utilisent un ou plusieurs LGRB. D'après le panel, Zotero est très largement utilisé : presque $89 \%$ des documentalistes et bibliothécaires de l'enseignement supérieur déclarent y avoir recours.En complément et dans les

\footnotetext{
17 Voir l'article Framasoft sur Pybliographer: http://www.framasoft.net/article4108.html
} 
mêmes conditions qu'en SHS ou sciences dures, ils tirent profit d'EndNote $(40,74 \%)$ ou Mendeley (presque $26 \%$ ). Cela tend à confirmer que Zotero est surtout appréciépour la détection de notices, mais que la gestion de la bibliographie est plutôt confiée à un logiciel tiers dédié à cet usage. En effet, seuls $20 \%$ de la tranche que nous étudions exploitentZotero sans logiciel complémentaire.

\section{Résultats et discussion}

Grâce à l'interprétation de cette collection de données, nous allons établir des profils techniques d'utilisateurs de systèmes d'informations documentaires. Ces profils permettront ultérieurement de définir un cahier des charges pour la modélisation de SRI par population.

\section{Profil sciences humaines et sociales}

En SHS, le profil technique type est très majoritairement caractérisé par l'usage d'un traitement de textes. Une faible proportion s'oriente,avec l'expérience, vers le compilateur. Pour ce qui est des usages typiquement liés à la bibliographie, une moitié intègre les citations et rédige sa bibliographie manuellement. Cela est en corrélation avec le fait que moins d'un sondé en SHS sur deux connaisse au moins un format de fichier bibliographique.Les formatsXML OpenDocument et RIS sontemployés chacun par presque $10 \%$ de l'échantillon en SHS. Pour la gestion de la bibliographie, seule une moitié des usagers utilise une assistance logicielle dédiée à cet usage (LGRB). Parmil'autre moitié,20 \% se servent des fonctionnalités intégrées au traitement de texte. Cependant,le plus souvent, les éléments bibliographiques sont intégrés et gérés sans assistance logicielle dans les documents.

Pour ceux qui recourent à un LGRB en sciences humaines et sociales, la pratique la plus courante est de s'appuyer surZotero pour la détection de notices et de l'associer à Mendeley ou EndNote pour la gestion.

Profil sciences dures

En sciences dures,les compilateurs de texte de type TeX sont largement connuspour la production de documents scientifiques. Ces outils sont majoritairement utilisés dès la fin de la thèse. Cela induit le choix de BibTeX comme format de bibliographie dans la plupart des cas.

Pour gérer leur bibliographie, les chercheurs en sciences dures exploitent pour plus de la moitié d'entre eux un logiciel dédié, souvent JabRef ou Mendeley en collaboration avec Zotero. Pour rappel, l'usage conjointde Zotero avec un autre outil permet de repérer et d'enregistrer les notices bibliographiques depuis Internet puis de gérer la bibliographie avec un logiciel de bureau.

Le profil documentaliste (ou bibliothécaire)

Les documentalistes et bibliothécaires dans l'enseignement supérieur utilisentpresque exclusivement un traitement de texte pour leur production écrite. Ils préfèrentmajoritairement les formats BibTeX et RIS qu'ils génèrent depuis Zotero et gèrent avec EndNote ou Mendeley.

\section{Conclusion et perspectives}

Il faut retenir de cette étude que, dans l'enseignement supérieur, les technologies dédiéesà la gestion bibliographique en ISTne bénéficient pas encore d'une large diffusion.

Dans les sciences dures, les contraintes éditoriales induisent un recours à des formats spécifiques. Ces usages sont facilités par l'emploi de logiciels dédiés. Pour cette raison, 
les LGRB se sont tout naturellement imposés pour un chercheur sur deux en sciences dures.En sciences humaines et sociales, les pratiques sont plus éparses du fait de pratiques éditoriales institutionnelles moins rigides.L'usage de l'assistance numérique à la gestion de références bibliographiques ne semble pas être la norme. L'adoption des outils intégrés au traitement de texte ne s'est pas non plus imposée comme la solution idéale. La constitution de bibliographie reste donc " artisanale », ce qui peut s'expliquer par le fait que ces fonctionnalités sont relativement récentes.

Il est évident qu'une bonne connaissance des outils, normes et formats utilisés par les usagers de l'information est un prérequis pour modéliser un SRI compatible avec la population cible. Ainsi, on n'envisagera pas un outil de recherche d'IST en sciences humaines de la même manière qu'un SRI en sciences dures, les formats et styles bibliographiques étant différents. Cependant, la constante dans tous les cas est que l'information se doit d'être visible et exportable, mais aussi compatible avec les outils répandus de glanage contextuel d'information comme Zotero. Chaudiron et Ihadjadene préconisaient à ce propos "d'intégrer dans la réflexion les mutations techniques des dispositifs de production, de recherche, de partage et de diffusion de l'information »[4].

Il est à prévoir dans les années à venir que ces pratiques de recherche d'IST, actuellement intégrées au cursus universitaire grâce au $\mathrm{C} 2 \mathrm{i}^{18}$, se propageront dans le monde de l'enseignement supérieur et de la recherche. En effet, les modules D4 du « C2i étudiant» et $\mathrm{A} 2 \mathrm{du}$ "C2i enseignant» rendent obligatoire l'apprentissage de ces fonctionnalités avancées depuis 2012[6].Il est donc vital que les SRI d'aujourd'hui et de demain soient pensés pour répondre aux besoins induits par ces nouvelles pratiques. Les éditeurs scientifiques, les moteurs de recherche scientifique et les archives numériques ouvertes ont déjà adapté leurs interfaces en les enrichissant de métadonnées au format « web de données ». De plus, l'ergonomie des SRI se doit d'aider les usagers à faire face à la surcharge cognitive due à l'afflux informationnel et l'orienter vers un choix adapté. Certains acteurs du domaine de l'IST ont commencé à offrir des moteurs de recommandation pour répondre à ce besoin. Qu'en est-il des OPAC des bibliothèques de l'enseignement supérieur sur ces deux points ?

Jusqu'à présent, comme le montre l'étude, les LGRB étaient principalement utilisés par les professionnels de la recherche d'informations et par les chercheurs en STM, familiers des interfaces informatiques. Ces logiciels souffraient d'une interface "pensée par des informaticiens pour des informaticiens ». Cela excluait les chercheurs en SHS qui ne les employaient pas, faute de pouvoir les installer et les utiliser simplement. Cette tendance s'inverse depuis peu, avec des outils plus simples à installer et qui communiquent intuitivement avec le traitement de texte. Cette appropriation des logiciels de gestion de références bibliographiques par les enseignants chercheurs va-t-elle impacter les usages des documentalistes et bibliothécaires, principalement ceux qui exercentdans le milieu de l'enseignement et de la recherche?

\section{Bibliographie}

[1]AMERICAN PSYCHOLOGICAL ASSOCIATION.Publication manual of the American Psychological Association, 6 ${ }^{\text {th }}$ ed., 2d print. Washington DC: American Psychological Association, 2010

[2] ANDREU R., RICART i COSTA J., VALOR J. Information Systems: Strategic Planning: a source of competitive advantage. NCC Blackwell, 1992

18 Le Certificat Informatique et Internet (C2i) atteste de compétences théoriques et pratiques des étudiants dans les nouvelles technologies. 
[3] BERMÈS E., MARTIN F. " Le concept de collection numérique ». Bulletin des

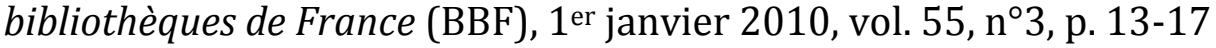

[4] CHAUDIRON S., IHADJADENE M. « De la recherche de l'information aux pratiques informationnelles ». Études de communication, 2010, vol. 35, n², p. 13-30

[5] GANDON F. "SparQL et la recherche dans le web de données ». DocumentalisteSciences de l'information, décembre 2011, vol. 48, n 4, p. 35

[6] HETZEL P. "Création du C2i niveau 2 "enseignant" et modalités de délivrance ». Bulletin officiel de l'enseignement supérieur, février 2011, p. 8

[7] HEURGON E. « Urbanisme et architecture des systèmes d'information ». Technologies de l'informatique et société, 1990, vol. 2, n³, p. 39-55

[8] KAENEL (de) I., IRIATRE P. « Les catalogues des bibliothèques : du web invisible au web social. ». Revue électronique suisse de science de l'information (RESSI), 2007, n5, http://archivesic.ccsd.cnrs.fr/sic 00141660

[9] KÉFI H., KALIKA M. « Le cadre analytique structurationniste de l'évaluation des SI ». In:Évaluation des systèmes d'information: une perspective organisationnelle. Paris :Economica, 2004. p. 18

[10] MASUR R. Étude sur la valorisation des logiciels de gestion de références bibliographiques (LGRB) dans le milieu des bibliothèques universitaires romandes, et sur les avantages et limites du plug-inZotero. Genève: Haute école de gestion de Genève (HEG-GE), 2009, 189 p., http://doc.rero.ch/lm.php?url=1000,41,9,20091112142141PA/TDB 2101.pdf

[11] POUPEAU G. " Microformats, RDFamicrodata: représenter le contenu d'une page web ». Documentaliste-Sciences de l'information, décembre 2011, vol. 48, n², p. 33

[12] POUYLLAU S. " Les moteurs de recherche profitent aussi de la sémantique ». Documentaliste-Sciences de l'information, décembre 2011, vol. 48, n² 4, p. 36-37

[13] RANJARD S. "Pratiques et attentes des publics des médiathèques ». Bulletin des bibliothèques de France, janvier 2000, vol. 45, n5, p. 102-107

[14] RIPON R., EVANS C. « La mise en œuvre d'une étude quantitative par questionnaire : vices et vertus du chiffre ». In:Mener l'enquête. Guide des études de publics en bibliothèque. Villeurbanne: Presses de l'Enssib, 2011, p. 6279,http://www.lcdpu.fr/livre/?GCOI=27000100949520

[15] ROUQUETTE M. LaTeX appliqué aux sciences humaines. Tampere (Finlande) :Atramenta, 2012. 270 p., http://www.atramenta.net/books/latex-scienceshumaines $/ 79$

[16] RUIZ C. N. et J. "Vers une conception globalisée des systèmes d'information intégrant tous leurs usages ». La Revue des sciences de gestion, 2007, vol. 223, n¹, p. 8797.

[17] VASSILIADIS P. " A survey of Extract-Transform-Loadtechnology ». International Journal of Data Warehousing and Mining (IJDWM), 2009, vol. 5, n³, p. 1-27

[18] WAHNICH S. « Enquêtes quantitatives et qualitatives, observation ethnographique ». Bulletin des bibliothèques de France, janvier 2006, vol. 51, n6, p. 8-12

[19] ZWEIFEL C. « Logiciels de gestion de références bibliographiques: citons le libre! ». Revue électronique suisse de science de l'information (Ressi), mai 2008, $\mathrm{n}^{\circ} 7$, http://campus.hesge.ch/ressi/Numero 7 mai2008/articles/HTML/RESSI 045 Zweifel.htm 